\title{
Automated assessment of serial SPECT myocardial perfusion images
}

\author{
Ami E. Iskandrian, MD, ${ }^{\mathrm{a}}$ Ernest V. Garcia, PhD, ${ }^{\mathrm{b}}$ Tracy Faber, PhD, ${ }^{\mathrm{b}}$ and \\ John J. Mahmarian, MD $^{c}$
}

\section{See related article, doi: 10.1007/s 12350-008-9018-0}

Investigations during the first 2 decades of modern nuclear cardiology focused on methodological refinements and establishing a solid foundation for myocardial perfusion imaging (MPI) as a reliable method for detecting coronary artery disease (CAD). The 2 decades after that emphasized the prognostic value of the test in virtually every group of patients and also challenged the prevailing wisdom that coronary angiography is the undisputed "gold standard" by showing that a physiological assessment is even more meaningful than anatomic descriptors when predicting the outcome of CAD patients. ${ }^{1}$

As gated SPECT became widely accepted in mainstream cardiology, additional uses emerged. Leading the list (because of its clinical, research and regulatory implications) was the question of how best to assess changes between serial images and interpret their clinical significance. Could serial imaging be used to assess progression or regression of left ventricular (LV) ischemia and thereby dynamically track risk, define the impact of a novel or old anti-ischemic therapy, or meet the standards of the Food and Drug administration (FDA) for approval of a new therapy, imaging agent, or stress agent? ? $^{2-5}$

The basic argument is not really whether quantitative analysis is needed to assess such serial changes in perfusion, but whether an automated program or a semiquantitative visual analysis should be routinely utilized. Our field has many examples that attest to the merits of

From the Department of Medicine, ${ }^{a}$ University of Alabama at Birmingham, Birmingham, AL; Department of Radiology, ${ }^{\mathrm{b}}$ Emory University, Atlanta, GA and The Methodist DeBakey Heart and Vascular Center, ${ }^{\mathrm{c}}$ The Methodist Hospital, Houston, TX.

Reprint requests: Ami E. Iskandrian, MD, The Department of Medicine, University of Alabama at Birmingham, 318 LHRB, 1900 University BLVD, Birmingham, AL 35294; aiskand@uab.edu, aiskand@cardmail.dom.uab.edu.

J Nucl Cardiol 2009;16:6-9

$1071-3581 / \$ 34.00$

Copyright $(\subseteq) 2008$ by the American Society of Nuclear Cardiology. doi:10.1007/s12350-008-9020-6 automated analysis by gated SPECT for measuring many indices of LV function such as ejection fraction (EF), volumes, wall motion and thickening, transient dilation, and the more recently described indices of dyssynchrony by phase analysis. ${ }^{1,6}$ This discussion, however, concerns perfusion images.

Despite the digital nature of SPECT images, interpretation has been, for the most part, based on a visual assessment, such as normal or abnormal-and if abnormal, scar, ischemia, or both.

Most studies in the literature assessing the diagnostic and/or prognostic accuracy of perfusion imaging have largely relied on visual analysis using SSS (summed stress score), SDS (summed difference score), and SRS (summed rest score). ${ }^{1}$ These methods have worked well because they are based on interpretation of a single set of images and probably because patients' outcomes or clinical diagnoses are not readily affected by slight inaccuracies.

However, when two sets of images are serially compared there arise multiple challenges. Is there improvement or worsening in the extent and severity of LV hypoperfusion and/or ischemia, and how much and where? Were the acquisition parameters the same for both image sets including the stressor modality and the type/dose of radiopharmaceutical administered? Is the image quality the same and how does that impact the observed changes? Should the images be read randomly or side-by-side, blinded or un-blinded, by consensus or separately? It is very likely that a simple definition of normal versus abnormal, or small, medium, and large will not be sufficient to answer these questions, and hence, a more "quantitative" method is required. $^{2,7}$ A brief summary might be helpful at this point.

\section{QUANTITATIVE METHODS FOR ASSESSING PERFUSION}

There are two semi-quantitative methods used to assess myocardial perfusion studies: visual and automated. Either method incorporates the standard 17-segment LV anatomic model to derive SSS, SDS, and SRS, although the visual method is more often used. 
These summed scores can be converted to \% abnormality of the LV myocardium by dividing the derived number by the highest potential abnormal score. The SSS is a composite score that reflects the extent and severity of LV hypoperfusion but does not take into account the vascular location of the abnormality. Thus an identical SSS could be derived from a large but mild abnormality versus a severe but small abnormality. Similarly, the same SSS before and after an intervention could represent resolution of an abnormality in one vascular territory and the development of a new abnormality in another. ${ }^{1}$

The alternative is to use "polar maps", which express the observed extent of stress-induced LV hypoperfusion as a \% of LV myocardium along with the extent of scintigraphic scar and ischemia. Like the SSS, polar maps provide both a regional and global assessment of the extent and severity of the perfusion abnormality in each of the three vascular territories. However, with polar plot quantitative analysis, the individual interpreter variability associated with calculating a SSS is removed, theoretically leading to a more reproducible assessment of serial images. SSS measurements have some intrinsic limitations when used for evaluating changes, related to their rather low number of discrete segments (i.e., 17) and total possible scores. For example, even a small rotation of the left ventricle between serial studies may cause the defect to move with respect to the segment boundaries, and thus change the SSS. A polar map-based approach will be less sensitive to this.

Nevertheless, the global indices (SSS and polar maps) have yielded powerful outcome data that have stood the test of time and therefore are very meaningful in patient care. ${ }^{4}$

There are multiple software packages that allow image interpretation in the fashion discussed above, and in general, are all based on circumferential profile analysis. The three most commonly available commercial ones are QPS (Cedars Sinai), Emory tool box (Emory), and 4D-MSPECT (Michigan). Other programs include those introduced by the investigators at Yale, Mayo, and Charlottesville, to mention a few. These various software programs differ from each other in many other ways including the analysis of circumferential profiles, slice selection, slice thickness, number of slices analyzed, the threshold for an abnormality (i.e., SDs above/below normal pixel values) and normal databases used for comparison, and methodology for quantifying ischemia (i.e., changes in individual pixel count activity from stress to rest images).

These packages have been with us for a long time, but a precise estimate of how widely each is used (outside the institution where each is developed) is not available because of the fact that the mere possession does not translate into use, or at least routine use.

\section{CLINICAL AND REGULATORY ISSUES IN IMAGE INTERPRETATION}

The ideal method for day-to-day image interpretation is the routine use of both visual and quantitative approaches as some artifacts are not recognized by the automated programs. Visual assessment of the raw image data allows identification of artifacts which may result in inaccurate quantification based on inconsistencies between the patient and the normal database. However, regardless of the method used (visual or quantitative), the study results will be heavily dependent on image quality and adherence to protocol. This is particularly important in multi-center trials when performing serial imaging. One would assume that after 40 years, image quality would be the same everywhere. This is the furthest from the truth due to multiple technical issues such as considerable hepatic and bowel activity from poorly timed image acquisition in patients receiving $\mathrm{Tc}-99 \mathrm{~m}$ labeled radiotracers, motion artifacts which are only variably correctable with software algorithms, and considerable image noise where large patient receives inadequate radiotracer doses. If four sets of images are acquired in a patient (stress/rest done at two different times), it only takes one of the four set of images to be of suboptimal quality to alter interpretation concordance. It also goes without saying that the highest agreement rates in image interpretation are in patients with normal images, and hence over-population of any trial by normal subjects could make any method (or reader) look better than actual performance.

The plethora of published papers based on visual analysis (and the inconsistent methodology of quantification among software products) is a factor as to why many users are reluctant to use automated programs, and why the FDA has insisted on visual analysis. The FDA often requires three independent readers (not even consensus reading) and then examines the interpretation by each reader, the median, or the average. ${ }^{5}$

Unlike blinded image reading currently required to satisfy the FDA, in clinical use the reader has the luxury of side-to-side comparison of images, which in our opinion is ideal because the reader can adjust for inconsistency in image quality. In addition, changes in LV orientation, shape, or size could be accounted for, and in some cases adjusted with reprocessing. We routinely display serial images on the same screen to assess location, severity, and extent of perfusion abnormalities. This must be at least a secondary end-point in any big study design or trial. 


\section{CURRENT STUDY}

In this issue of the journal, a well-respected group in our field which pioneered the development of the semi-quantitative SSS, concludes that quantitation by an automated method is more reproducible than the standard visual method for determining the extent of perfusion abnormality when image quality is good to excellent on both studies $(87 \%) .{ }^{8}$ Further, Berman et al predict that automated methods should be superior to visual methods in randomized clinical trials and when monitoring the effects of therapy in an individual patient. ${ }^{8}$ Despite the limitations of the study, we concur with the overall conclusions, and our own experience is in accordance with the findings. The limitations of the study are partly addressed by the authors and include the small sample size (only 25 patients with ischemia), the long time intervals between the two studies, and the fact that all studies were from same institution. Their conclusions might have been different if studies of varying image quality were included or if patients with more severe perfusion defects, dilated LV, and thinned out myocardium had been included. These issues need further study. For the time being, we would like to focus on three issues: the method of automated analysis, the implications in interpretation of serial testing, and the impact on sample size calculation.

Current automated quantitative analysis methods deal with each set of images (first and second sets of stress/rest) as independent entities, and for that matter they are not treated differently if the first set was from patient $\mathrm{A}$ and the second set from patient B. Newer software programs could do better by comparing stress 1 versus stress 2 and rest 1 versus rest 2 , plus stress versus rest in sets 1 and 2. Advanced programs could adjust for LV size and position, a method referred to as "warping". The aim is to derive a score on a pixel-by-pixel basis between two sets of images, and the score might be positive or negative depending on whether there is improvement or worsening. In fact, this technical development has moved ahead of clinical applications, and we just need to test these newer programs prospectively.

Berman et al found that the estimated thresholds for a real patient change beyond variability for stress, rest, and ischemic defects to be $<7 \%$ by the automated method and $10 \%-13 \%$ by the visual method. ${ }^{8}$ Assuming one uses an automated method for serial testing in a clinical trial, what type of patients should be recruited given these thresholds, or alternatively, are these thresholds applicable to all patients regardless of size of baseline abnormality? One option is to express the change from absolute to relative percentages as is often done in large trials in acute myocardial infarction or acute coronary syndromes. Thus a change from $30 \%$ to $15 \%$ and a change from $10 \%$ to $5 \%$ both represent a $50 \%$ reduction and both are meaningful, even though the change in the latter patient might be within the error of measurement. Otherwise, we cannot study or recruit patients with small defects at entry! Unrealistic, as most of our patients are in this category. ${ }^{9}$

Another issue is that no study has reported the reproducibility of serial imaging using automated quantitative analysis when combined with attenuation correction, which is ironic given the fact that attenuation correction has been around for some time, and its value in single sets of studies (accuracy and outcome) have been reported. Attenuation correction would be expected to decrease image pixel heterogeneity and thereby reduce the error of the estimate. Whether this would improve the accuracy and/or reproducibility of the perfusion defect measured remains to be seen. ${ }^{10}$

Lastly, the impact on sample size must be considered. The lower estimated thresholds by the automated method would substantially reduce the number of patients needed to be enrolled in a clinical trial, thereby expediting trial completion and saving costs to the sponsor. Not only incorporating quantitative analysis into our routine imaging armamentarium, but also standardizing and optimizing our current methodology for quantification should be our ultimate goal. ${ }^{11}$

In conclusion, automated assessment is superior to visual assessment of MPI and should be adopted in future studies and clinical trials that involve assessment of more than one set of images per patient.

\section{References}

1. Iskandrian AE, Garcia EV, editors. Nuclear cardiac imaging: Principles and applications. 4th ed. Oxford: Oxford University Press; 2008.

2. Zoghbi GJ, Dorfman TA, Iskandrian AE. The effects of medications on myocardial perfusion. J Am Coll Cardiol 2008;52:401-16.

3. Mahmarian JJ, Moye LA, Verani MS, et al. High reproducibility of myocardial perfusion defects in patients undergoing serial exercise thallium-201 tomography. Am J Cardiol 1995;75:1116-9.

4. Mahmarian JJ, Shaw LJ, Filipchuk NG, et al. A multinational study to establish the value of early adenosine technetium-99m sestamibi myocardial perfusion imaging in identifying a low-risk group for early hospital discharge after acute myocardial infarction. J Am Coll Cardiol 2006;48:2448-57.

5. Cerqueira MD, Nguyen P, Staehr PS, Underwood SR, Iskandrian AE, On behalf of the ADVANCE-MPI Trial Investigators. Effects of age, gender, obesity, and diabetes on the efficacy and safety of the selective A2A agonist Regadenoson versus adenosine in myocardial perfusion imaging: Integrated ADVANCE-MPI trial results. J Am Coll Cardiol Imaging 2008;1:307-16.

6. Trimble MA, Borges-Neto S, Velazquez EJ, et al. Emerging role of myocardial perfusion imaging for cardiac resynchronization therapy. Am J Cardiol 2008;102:211-7. 
7. Iskandrian AE, Garcia EV, Faber T. Analysis of serial images: A challenge and an opportunity. J Nucl Cardiol 2008;15:23-6.

8. Berman DS, Kang X, Granary H, et al. Quantitative assessment of myocardial perfusion abnormality on SPECT myocardial perfusion imaging is more reproducible than expert visual analysis. J Nucl Cardiol. doi:10.1007/s12350-008-9018-0.

9. Shaw LJ, Berman DS, Maron DJ, et al. Optimal medical therapy with or without percutaneous coronary intervention to reduce ischemic burden: Results from the clinical outcomes utilizing revascularization and aggressive drug evaluation (COURAGE) trial nuclear substudy. Circulation 2008;117:1283-91.

10. Singh B, Bateman TM, Case JA, Heller GV. Attenuation artifact, attenuation correction, and the future of myocardial perfusion SPECT. J Nucl Cardiol 2007;14:153-64.

11. Gibbons RJ, Valeti US, Araoz PA, Jaffe AS. The quantification of infarct size. J Am Coll Cardiol 2004;44:1533-42. 